\title{
LHCD during current ramp experiments on Alcator C-Mod
}

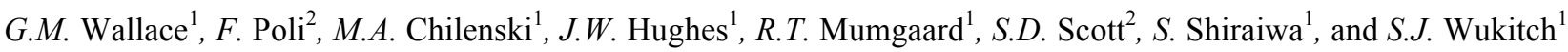 \\ ${ }^{1}$ MIT Plasma Science and Fusion Center, Cambridge, MA 02139 USA \\ ${ }^{2}$ Princeton Plasma Physics Laboratory, Princeton, NJ 08543 USA
}

\begin{abstract}
The lower hybrid current drive (LHCD) system on Alcator C-Mod is capable of sustaining fully non-inductive discharges for multiple current relaxation times $\left(\tau_{c r} \sim 200 \mathrm{~ms}\right)$ at line averaged densities in the range of $5 \times 10^{19} \mathrm{~m}^{-3}$. Some of these non-inductive discharges develop unstable MHD modes that can greatly reduce current drive performance, particularly in discharges with plasma current of $0.5 \mathrm{MA}$ or less $[1,2]$. Avoiding these unstable MHD modes motivated an experiment to test if the stable current profile shape of a higher current non-inductive discharge could be achieved in a lower current discharge. Starting from a discharge at $0.8 \mathrm{MA}$, the plasma current was ramped down to $0.5 \mathrm{MA}$ over $200 \mathrm{~ms}$. The surface voltage of the plasma swings negative during the ramp, with the loop voltage reversal impacting the edge fast electron measurements immediately. Little change can be seen during the $I_{p}$ ramp in the core fast electron measurements, indicating that the loop voltage reversal does not penetrate fully to the magnetic axis on the timescale of the current ramp. The resulting discharge did not exhibit deleterious MHD instabilities, however the existence of this one discharge does not necessarily represent a robust solution to the problem.
\end{abstract}

\section{Introduction}

Lower hybrid current drive (LHCD) is capable of sustaining fully non-inductive discharges for multiple current relaxation times $\left(\tau_{c r} \sim 200 \mathrm{~ms}\right)$ at line averaged densities in the range of $5 \times 10^{19} \mathrm{~m}^{-3}$ on Alcator C-Mod. CMod discharges with strong off-axis current drive $\left(\frac{\Delta V}{V}>\right.$ $0.5)$ sometimes develop MHD instabilities as the current profile evolves. These instabilities can be benign, but often result in loss of current drive efficiency and degraded transport [1]. Figures 1 and 2 show an example of a discharge with MHD instability developing around $1.15 \mathrm{~s}$.

A database of existing discharges with lower hybrid current drive (LHCD) has been created. The database includes information on net $\mathrm{LH}$ power, launched $\mathrm{n}_{\|}$, plasma current, loop voltage, electron density and temperature, $\mathrm{Z}_{\mathrm{eff}}$, driven current, 0-D LHCD efficiency, and the presence of deleterious MHD instabilities. The range of parameters for which MHD is problematic is shown in Figure 3. MHD instabilities also appear to be responsible for termination of $T_{e}$ ITBs in reverse shear discharges [2]. Although the MHD activity typically causes a decrease in current drive efficiency, some discharges with MHD continue to show good current drive efficiency as shown in Figure 3.

The goal of this experiment was to better understand how to avoid these instabilities in discharges with significant off-axis current drive. To that end, upgrades to the motional stark effect (MSE) diagnostic improve accuracy and time resolution of current profile reconstructions for 2015 [3] for input to MHD stability calculations.

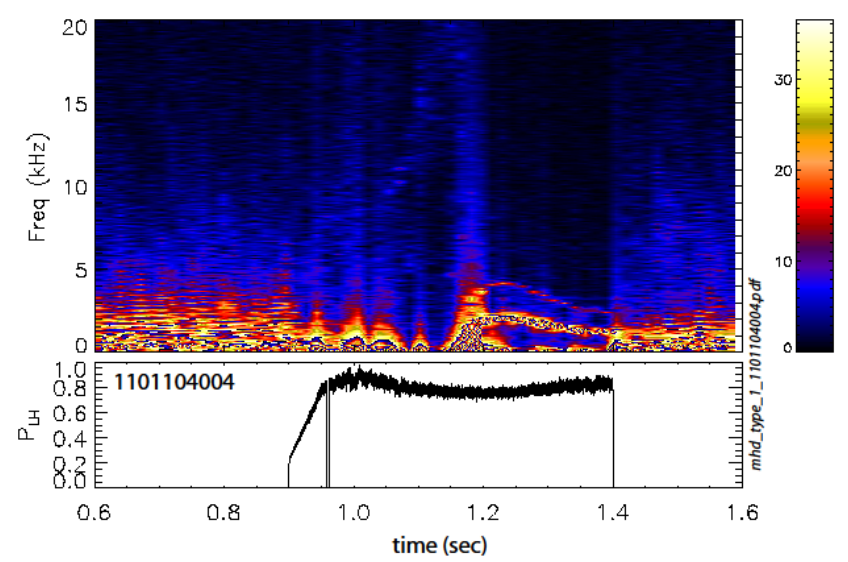

Fig. 1. Low-frequency $(<\sim 5 \mathrm{kHz}) \mathrm{MHD}$ is sometimes observed on magnetic pickup loops (shown above) and soft x-ray arrays during high-power LHRF in Alcator C-Mod. The onset occurs just before $1.2 \mathrm{~s}$ in the discharge shown.

\section{Experimental approach}

Experiments on Tore Supra [4] and ASDEX [5] have identified certain "pre-forming" techniques to eliminate the occurrence of MHD instabilities during LHCD. Some of the techniques identified are: step changes in $P_{L H}$ during the discharge; ramping down $I_{p}$ after turning on the LHRF; and intentional distortion of the $n_{\|}$spectrum by

\footnotetext{
Corresponding author: wallaceg@mit.edu
} 
reducing the number of waveguide columns energized. The experiments on C-Mod focused on ramping $I_{p}$ during the LHRF pulse.

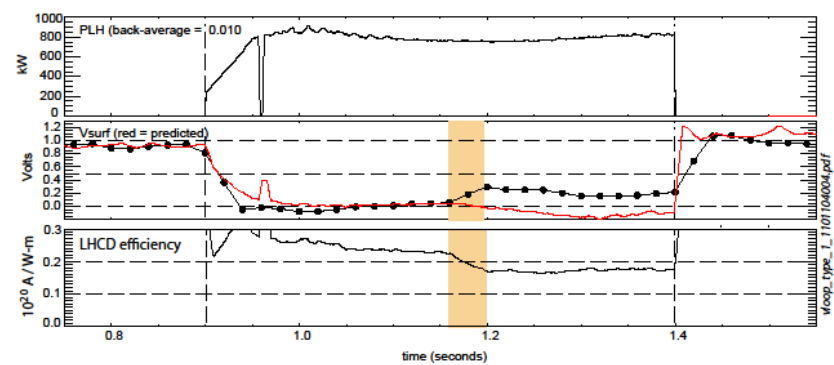

Fig. 2. Low-frequency MHD instabilities sometimes, but not always, results in a reduction in LH current-drive efficiency. The shaded region indicates the onset of low frequency MHD instability in this discharge.

Discharges at higher $I_{p}$ exhibit higher current drive efficiency and more off-axis current drive on C-Mod, while also less commonly suffering from low frequency MHD instabilties. Starting at higher $I_{p}$, then ramping the current down while LH is energized may help to keep a more stable, off-axis current drive profile while also increasing the fraction of power that is driven noninductively.

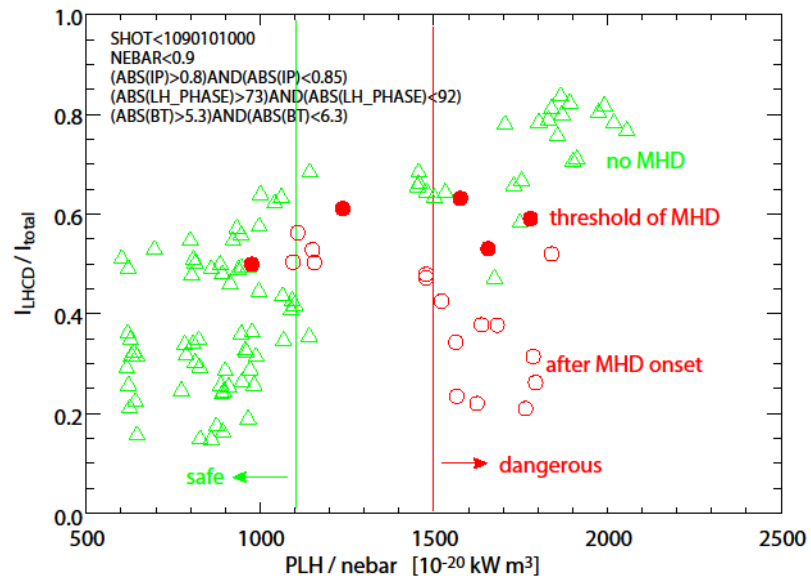

Fig. 3. The probability of deleterious MHD generally increases as the LHRF power normalized to line average density increases. Green triangles represent stable discharges. Solid red circles represent unstable discharges prior to onset of instability, and open red circles represent unstable discharges following onset of instability.

\section{Experimental results}

Experiments conducted in the final days of C-Mod operation explored the use of current ramps during the LHRF pulse to improve current drive performance and MHD stability. This paper will focus on shot 1160929029, an upper single null discharge with a current ramp from $0.8 \mathrm{MA}$ to $0.5 \mathrm{MA}$ during the LHRF pulse. Figure 4 shows some key plasma parameters from this discharge. The LHRF pulse began at $0.7 \mathrm{~s}$, with the $I_{p}$ ramping down between 0.85 and $1.05 \mathrm{~s}$. The LHRF pulse ended at $1.4 \mathrm{~s}$. LHRF power was $650 \mathrm{~kW}$ at a launched peak $n_{\|}$of 1.92. Plasma kinetic profiles measured before and after the current rampdown (Figure 5) show that the density and temperature both drop slightly across most of the plasma profile during the rampdown. The timing of the LHRF pulse and $I_{p}$ ramp allow for a current relaxation timescale $\left(\tau_{c r} \sim 0.2 \mathrm{~s}\right)$ to elapse following the end of the $I_{p}$ ramp.
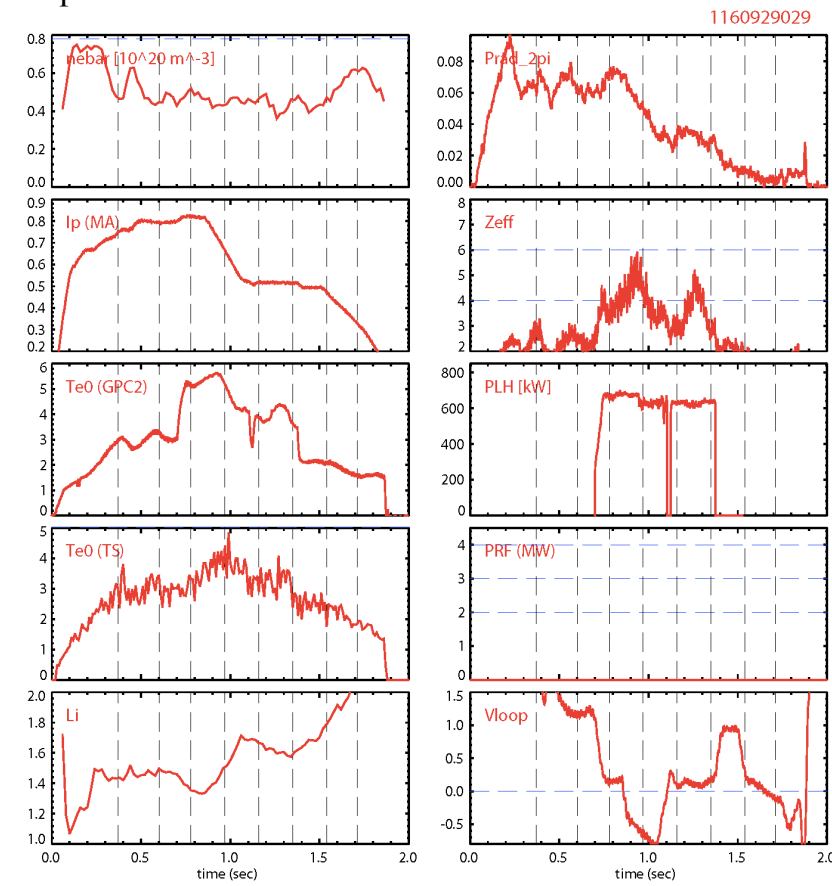

Fig. 4. Plasma parameters for discharge 1160929029. The plasma current ramps down from 0.8 to 0.5 MA during the LHRF pulse. Vertical dashed lines indicate MSE current profile measurement timepoints.

Prior to the current ramp, the loop voltage dropped from $\sim 1.2 \mathrm{~V}$ to $\sim 0.2 \mathrm{~V}$ during LHRF. As the current began to drop, the loop voltage swung to $\sim-0.7 \mathrm{~V}$ at $\sim 1.0 \mathrm{~s}$, then recovered back towards $\sim 0.1 \mathrm{~V}$ at the end of the $I_{p}$ ramp. Although the loop voltage was negative at the boundary of the plasma during this interval, the diffusion of the negative DC electric field into the plasma, and its effect on LH wave damping and current drive, must be considered through time-dependent simulations as the current diffusion time of $\sim 0.2 \mathrm{~s}$ is similar to the timescale of the loop voltage changes.

Figure 6 shows the MSE constrained EFIT current profile reconstructions from time slices during the Ohmic period, LHRF period before the $I_{p}$ rampdown, during the $I_{p}$ rampdown, and following the $I_{p}$ rampdown. The preLHRF current profile $(t=0.6 \mathrm{~s})$ was peaked with a central current density of $14 \mathrm{kA} / \mathrm{cm}^{2}$. As LHRF was applied to the $800 \mathrm{kA}$ segment of the discharge $(t=0.78 \mathrm{~s})$, the central current density dropped to $\sim 12.5 \mathrm{kA} / \mathrm{cm}^{2}$, with a corresponding increase in current density outside the midradius. As the current ramped down during the LHRF pulse $(t=0.96 \mathrm{~s})$, the central current density dropped sharply to slightly under $10 \mathrm{kA} / \mathrm{cm}^{2}$, with the current profile peaked just off-axis. The final MSE measurement occurred after the current ramp finished $(t=1.34 \mathrm{~s})$ and 
the discharge reached a new equilibrium. The final state of the current profile did not exhibit the off-axis peak seen during the current ramp, but does show some flattening of the current profile near the magnetic axis.
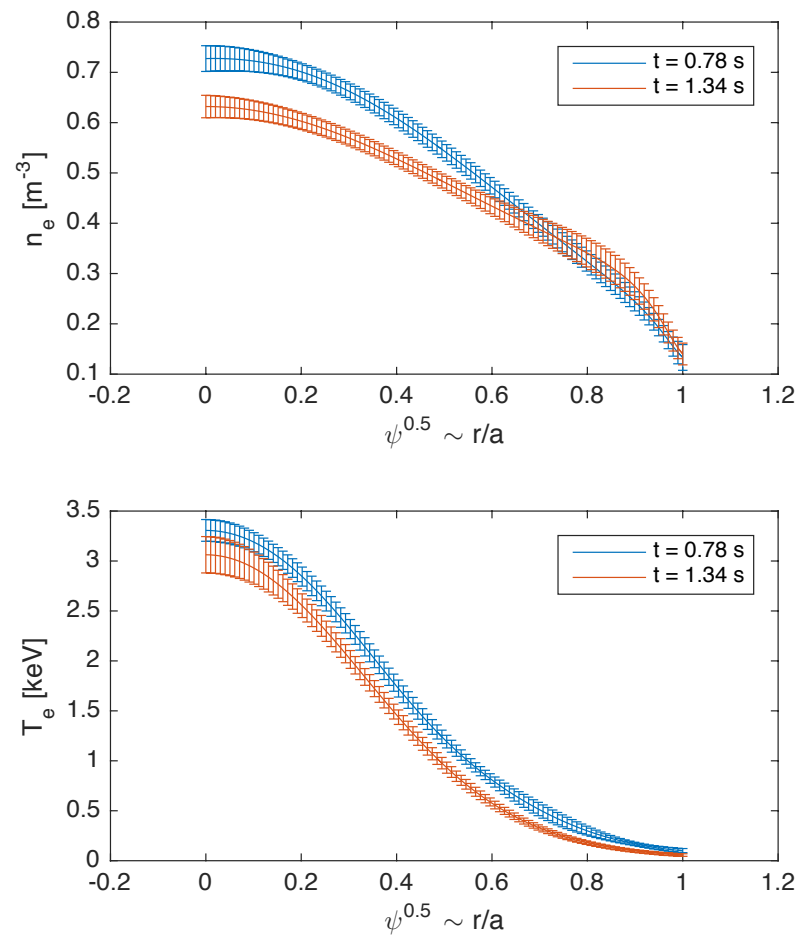

Fig. 5. Kinetic profiles measured by Thomson scattering for discharge 1160929029 before and after the current rampdown.

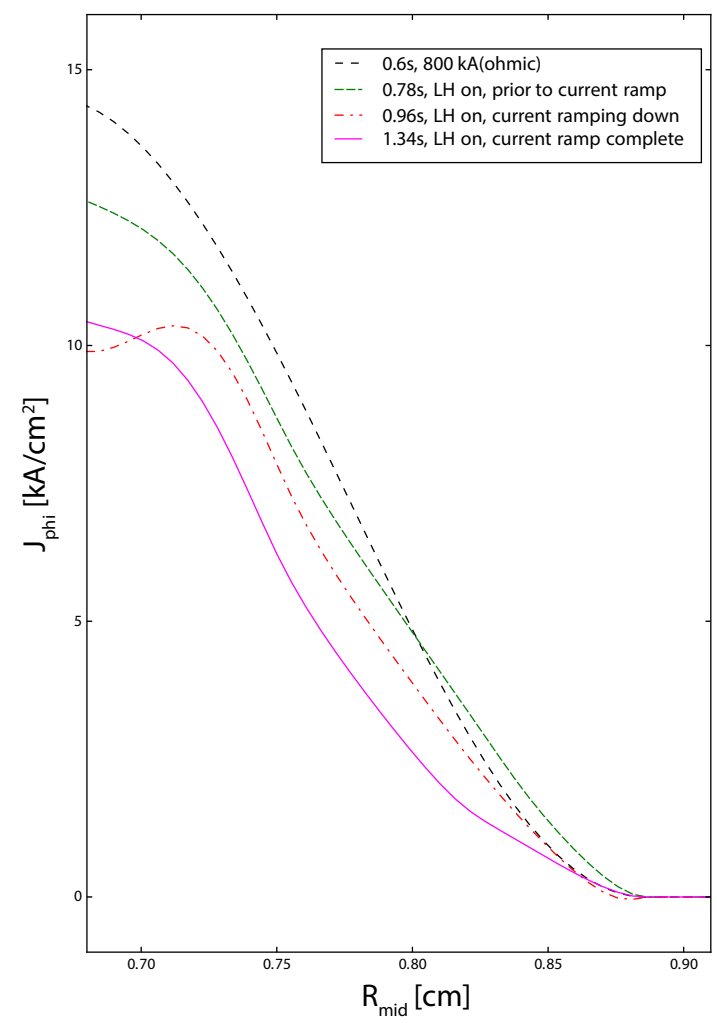

Fig. 6. MSE constrained EFIT current profile reconstructions of shot 1160929029.

Sawteeth in this discharge appear during the Ohmic period starting around $0.27 \mathrm{~s}$, and are suppressed shortly after the beginning of the LH pulse (before the start of the $I_{p}$ ramp). The safety factor profiles in Figure 7 shows weak shear reversal during the current rampdown, with a flat safety factor profile in the core during the low $I_{p}$ portion of the discharge.

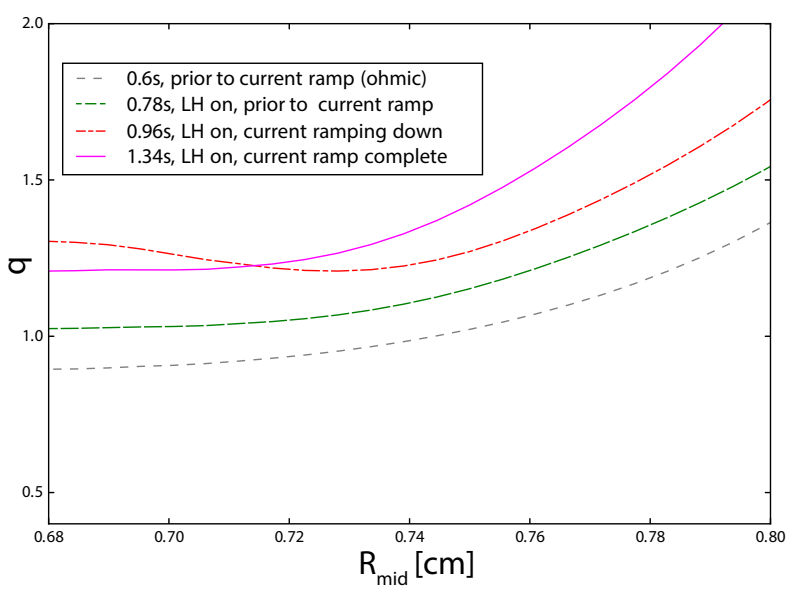

Fig. 7. MSE constrained EFIT safety factor profile reconstructions of shot 1160929029.

A short burst (0.99 to $1.02 \mathrm{~s})$ of MHD activity was observed during the current rampdown, but otherwise the discharge was free of the deleterious low frequency MHD instabilities shown in Figure 1.

A poloidally viewing hard $\mathrm{x}$-ray (HXR) camera is used to diagnose the non-thermal fast electron population for this discharge. Figure 8 shows the line integrated HXR emission profiles at the MSE measurement time points during the LHRF pulse. The HXR profile is considerably broader before the current rampdown as compared to after. The peak count rate in the plasma core does not change during the rampdown relative to the prerampdown value, indicating that the effect of the large negative loop voltage imposed at the edge of the plasma does not reach the core on the timescale of the ramp. Furthermore, the break in slop of the HXR emission plotted in Figure 9 occurs approximately $150 \mathrm{~ms}$ after the loop voltage at the plasma boundary changes sign, followed by a relatively modest reduction in HXR emission. This observation suggests that the externally applied toroidal electric field diffuses only to the outer regions of the plasma (HXR chords $<10$ and $>22$ ) on the timescale of the current rampdown. 

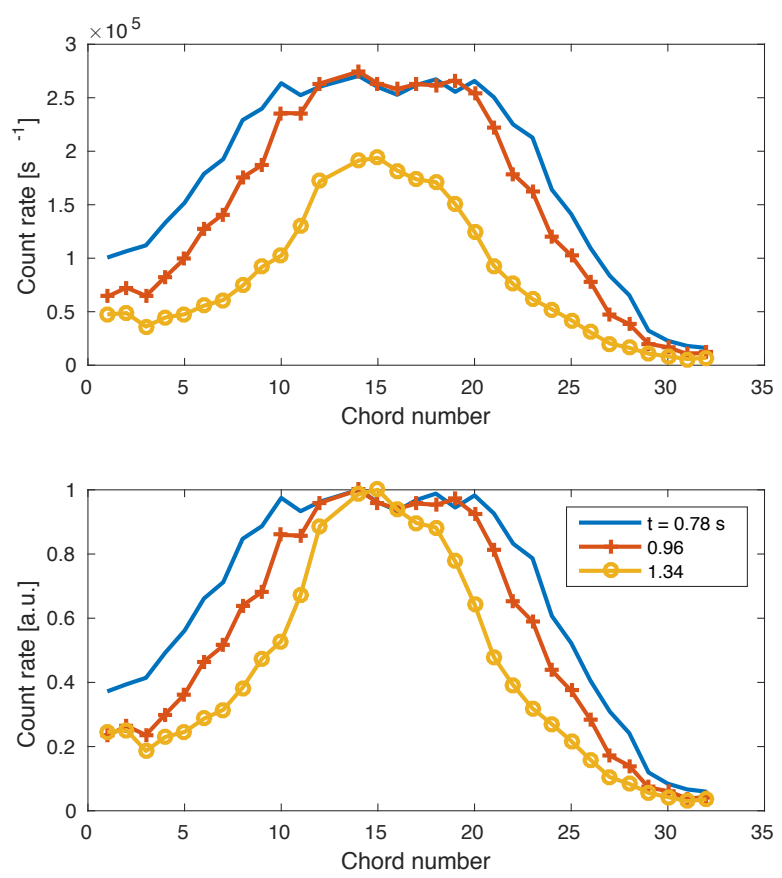

Fig. 8. Absolute (top) and normalized (bottom) hard x-ray emission profiles before, during, and after the current ramp.
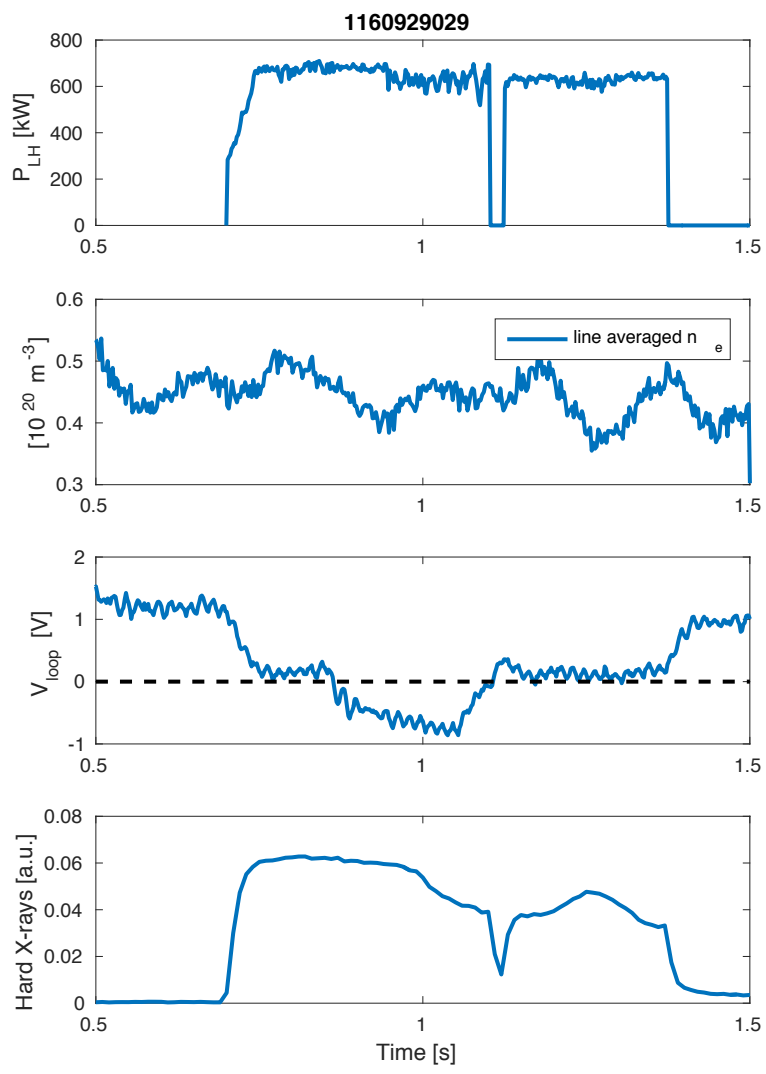

Fig. 9. Time history of net LHRF power, line averaged density, loop voltage, and hard x-ray count rate (chords 9-24).

\section{Discussion and conclusions}

The absence of MHD activity in this one discharge does not indicate that a solution to the problem of MHD instabilities reducing current drive efficiency has been identified, and more discharges are needed to increase statistical confidence. As Figure 3 shows, not every shot near full non-inductive current drive developed these instabilities, and additional discharges would be needed to determine if the technique is robust relative to $500 \mathrm{kA}$ discharges without the $I_{p}$ ramp. Further stability analysis on this discharge following the method presented in [6] will yield additional insight into whether the current ramp technique results in a more stable current profile.

Time-dependent analysis of this discharge using TRANSP [7] in conjunction with the GENRAY/CQL3D ray-tracing/Fokker-Planck simulation suite $[8,9]$ will be needed to determine the effect of the spatially and temporally varying toroidal electric field on wave damping and current drive. The use of GENRAY/CQL3D within TRANSP is described in [10], and a similar procedure will be followed in modelling this discharge.

This work was conducted on the Alcator C-Mod tokamak, an Office of Science user facility supported by US Department of Energy cooperative agreements DE-FC02-99ER54512 and DEAC02-76CH03073.

\section{References}

1. Scott, S., P. Bonoli, R. Mumgaard, S. Shiraiwa, G. Wallace, and D. Whyte. "Scaling of Global LHCD Efficiency in Alcator C-Mod." In APS Meeting Abstracts, vol. 1, p. 8021P. (2014).

2. S. Shiraiwa, et al. Nuc. Fus., Vol. 53, No. 11 (2013)

3. Mumgaard, R. T., S. D. Scott, and M. Khoury. "The multi-spectral line-polarization MSE system on Alcator C-Mod." Review of Scientific Instruments 87, no. 11 (2016): 11E527.

4. Zabiégo et al, Plasma Phys. Control. Fusion, 43 (2001) 1625-1639; Maget et al, Nucl. Fusion 47 (2007) 233-243

5. Zohm et al, PPCF, Vol. 33, No. 12, 1423-1441, 1991; Söldner et al, Nuc. Fus., Vol. 34, No. 7, 985-1016, (1994).

6. Ebrahimi, Fatima, A. Bhattacharjee, L. Delgado, S. Scott, J. R. Wilson, G. M. Wallace, S. Shiraiwa, and R. T. Mumgaard. "C-Mod MHD stability analysis with LHCD." In APS Meeting Abstracts. 2016.

7. Hawryluk, R. J. "An empirical approach to tokamak transport." Physics of plasmas close to thermonuclear conditions 1 (1980): 19-46.

8. A. P. Smirnov, R. Harvey, Bull. Am. Phys. Soc. 40 (1995) 1837.

9. R. W. Harvey, M. McCoy, The CQL3D FokkerPlanck Code, in: Pro- ceedings of the IAEA Technical Committee Meeting on Simulation and Modeling of Thermonuclear Plasmas, 1992, pp. 489 526.

10. F.M. Poli, et al. Plasma Physics and Controlled Fusion, Volume 58, Number 9 (2016). 